\title{
Intervention of the Occupational Therapist in the Oncological Patient with Cancer-Related Fatigue
}

\section{Fernandez Rodriguez EJ* and Sanchez Gómez C}

University of Salamanca, Spain

*Corresponding author: Eduardo Jose Fernandez Rodriguez, University of Salamanca, Spain,Email: edujfr@usal.es

\section{Clinical Note}

The occupational therapist as a health professional will carry out his intervention in all those patients who present a specific symptomatology or characteristics that require it. In the case of the cancer patient, which we are talking about, their intervention will not be based on directly attacking the neoplasm to prevent its development or to eliminate it, but will stick to acting as a member of the interdisciplinary work team, in charge of controlling secondary symptoms. To the oncological process, provide the necessary social support, both to patients and their caregivers, as well as to carry out actions that improve the quality of life of the individual.

In recent years, three phenomena have been expressed that make an intervention more and more essential from a rehabilitative point of view in this type of patient. These are:

- Increased incidence: According to Eurostat criteria, in the coming years, $42.5 \%$ of men will suffer from the disease; while in the case of women we are talking about $27.4 \%$.

- Increase in overall survival: Thanks to advances in diagnoses, treatments and preventive measures, in the last 20 years there has been a decrease of nearly $20 \%$ in mortality. Currently we find an average survival of close to $54 \%$ in oncological processes.

- Increase in symptoms secondary to oncological processes: As a consequence of this increase in incidence and this increase in overall survival, we find that these people receive more and more treatments, and as a consequence of this, their side effects increase. This, together with the side effects of the tumor processes themselves, means that we have to incorporate much more exhaustive measures that help control this secondary symptomatology expressed in patients [1-5].
The symptoms to which the occupational therapist will contribute to his improvement or adaptation of the cancer patient can be summarized as follows:

- Cancer-Related fatigue.

- Respiratory pathologies as a consequence of oncological processes: Dyspnea.

- Neurological pathologies as a consequence of oncological processes.

- Cognitive pathologies associated with oncological processes: chemobrain.

- Anxiety.

- Pain.

- Lymphedema.

All of them, without a correct performance, will generate dependency in the individual and will promote a significant deterioration in their autonomy, with their consequent deterioration in terms of quality of life [6].

Therefore, the role of occupational therapy in oncology will be to "rehabilitate and re-adapt an individual, with the aim of achieving their greatest functional, physical or cognitive performance, in their activities of daily living, regardless of their life expectancy" [7-9].

Due to the uniqueness and complexity of human occupation, each person diagnosed with cancer will experience different limitations in their occupations and restrictions on their community participation, which will vary throughout the course of the disease.

Cancer and its treatment can cause interruptions in daily routines that affect the way in which individuals carry out their self-care, their work, their leisure or their social 


\section{Annals of Physiotherapy \& Occupational Therapy}

activities. For example, people may experience difficulties with self-care activities, such as bathing or dressing. Others may experience difficulties performing essential job functions such as lifting, carrying, or having enough strength, both physically and cognitively, to perform a full-time job. Some people with cancer may experience difficulties with leisure activities, while others may experience difficulties in socializing with friends and/or family.

Individuals with cancer may experience these difficulties as a result of the disease or the effects of its treatment [10].

Common side effects of cancer or its treatment include fatigue, pain, weakness, cognitive difficulties, anxiety or depression, and changes in self-esteem or self-image. Occupational therapists address these effects through intervention aimed at restoring these functions. This will be done through the development of home exercise programs to improve strength and mobility, modifications in their daily activities, with energy saving techniques that people will carry out during important daily activities, or the modification of environments such as the place work, home or community [11-13].

\section{What is Occupational Therapy Intended for in the Patient?}

Teach the person to face their daily activities with their severely diminished functional reserves, in order to regain the ability to carry out their occupational roles within their potential margin.

It acts on the quality of life of the patient, since it improves their functioning at home and in the environment where they live. Patients report an improvement in their mood, feelings of optimism, self-control and self-esteem, with the consequent decrease in their anxiety [14].

The benefits of occupational therapy are appreciated in:

- The quality of life and the general well-being of the individual.

- The general state of health, both related to respiratory symptoms and tolerance to current exercise.

- Independence, the patient increases his autonomy and consequently his feeling of self-worth.

- The ability to perform their occupational roles in society. The performance of the occupational therapist will always be focused on complying with all the

\section{Principles of Continuous and Palliative Care, which are:}

- Comprehensive care of the individual.

- The patient and the family are the unit to be treated.

- Promotion of the autonomy and dignity of the patient.

- Active and rehabilitative therapeutic conception.

- Care objectives: well-being and quality of life.
- Importance of the environment or surroundings.

\section{Main Objectives of the Occupational Therapist in Oncology}

1. Promote the recovery, maintenance and compensation of functions and performance skills.

2. Sustain and promote the restoration and continuity of performance in occupational areas.

3. Promote the functional recovery of the individual.

4. Provide the adaptations and/or modifications necessary for the maintenance of the person's performance in their daily life.

5. Contribute to the improvement of the quality of life related to the health of the cancer patient through the correct control of present symptoms.

Next, we will analyze more deeply one of the symptoms, tumor asthenia, understanding its clinical characteristics, its form of evaluation, emphasizing the most appropriate measures or instruments from the perspective of the occupational therapist, and addressing it from a theoretical point of view, what would be the principles that would support their intervention [15].

\section{Cancer-Related Fatigue}

The National Comprehensive Cancer Network (NCCN) Fatigue Practice Guidelines Panel has defined CancerRelated fatigue as "a subjective feeling of fatigue related to cancer or its treatment, unusual, persistent, and interfering with normal activity".

Cancer-Related fatigue is the most common symptom associated with cancer and its treatment. The estimated prevalence varies between $60-90 \%$, depending on the diagnostic criteria used. Asthenia is considered the longestlasting and most disruptive symptom in cancer patients and has the greatest impact on quality of life parameters.

Is a frequent problem in oncology still undersized by professionals, who have polarized pain and relapse - free survival, despite the great impact here and all have for patients in their quality of life [16].

If we stress the term of tumor asthenia, that is, asthenia referred by oncological disease, we can find, in the 10th edition of the International Classification of Diseases (ICD10) the following diagnostic criteria for tumor asthenia.

\section{Icd-10 Criteria for Cancer-Related Astenia}

A. The following symptoms have been present every day or almost every day during the same two-week period in the 


\section{Annals of Physiotherapy \& Occupational Therapy}

last month:

Significant chronic fatigue, decreased energy, or increased need to lie down, out of proportion to any recent changes in activity level; in addition to five or more of the following:

1. Complaints of general weakness, heavy limbs.

2. Decreased concentration or attention.

3. Decreased motivation or interest in participating in regular activities.

4. Insomnia or hypersomnia.

5. Dream that does not refresh or restore.

6. Perception that you need to fight to overcome inactivity.

7. Marked emotional reactivity (for example, sadness, frustration, or irritability) in the face of chronic tiredness.

8. The difficulty of carrying out daily tasks is attributed to the feeling of tiredness.

9. Perception that there are problems with short-term memory.
10. Tiredness after strenuous exercise lasts for several hours.

B. Symptoms cause clinically significant impairment, or weakness, or impairment in social, professional, or other important behavioral areas.

C. There are signs in the medical history, medical examination, or test result that symptoms arise as a result of cancer or cancer treatment.

D. Symptoms do not arise primarily as a consequence of simultaneous psychiatric disorders, such as severe depression, somatization disorder, or delirium.

Cancer-Related fatigue is the most common symptom associated with cancer and its treatment. The estimated prevalence varies between $60-90 \%$, depending on the diagnostic criteria used. Asthenia is considered the most durable and disruptive symptom of cancer patients and has the greatest impact on quality of life parameters (Table 1).

\section{Ethiopathogeny of Cancer-Related Fatigue}

\begin{tabular}{|c|c|}
\hline \multicolumn{2}{|r|}{ Ethiopathogeny of Cancer-Related Fatigue } \\
\hline 1 & Cytokine production \\
\hline \multirow[t]{6}{*}{2} & Muscle disorders \\
\hline & Loss of muscle mass \\
\hline & Accumulation of lactic acid \\
\hline & Depletion of energy substances \\
\hline & Alteration in the distribution of muscle isoenzymes \\
\hline & Polyomyositis \\
\hline \multirow[t]{3}{*}{3} & Neurological disorders \\
\hline & Reticular formation dysfunction \\
\hline & Paraneoplastic syndromes \\
\hline \multirow[t]{6}{*}{4} & Metabolic and endocrinological disorders \\
\hline & Mellitus diabetes. \\
\hline & Hydroelectrolytic disorders \\
\hline & Hypothyroidism \\
\hline & Suprarrenal insufficiency \\
\hline & Hypopituitarism \\
\hline \multirow[t]{4}{*}{5} & Infections \\
\hline & Viral \\
\hline & Bacterial \\
\hline & Fungal \\
\hline 6 & Anemia \\
\hline \multirow[t]{2}{*}{7} & Specific treatments \\
\hline & Chemotherapy \\
\hline
\end{tabular}




\begin{tabular}{|c|c|}
\hline & Radiotherapy \\
\hline & Biotherapy. \\
\hline 8 & Symptomatic treatments \\
\hline & Opioids \\
\hline & Benzodiazepines \\
\hline & Antiemetics \\
\hline & Antihistamines \\
\hline 9 & Neuroleptics \\
\hline 10 & Overexertion \\
\hline 11 & Psychological disorders \\
\hline 12 & Comorbidity \\
\hline 13 & Tumor progression \\
\hline & Asthenia after treatment \\
\hline
\end{tabular}

Table 1: Aetiopathogenesis of tumor asthenia.

Evaluation instruments

Most used tumor asthenia evaluation instruments:

- PERFORM questionnaire.

- Piper Fatigue Scale (PFS).

- Visual Analog Scale (EVA).

- Functional Assessment of Cancer Therapy-Fatigue (FACT-F).

\section{Perform Questionnaire}

We consider this to be the ideal instrument to evaluate this symptomatic group. Until the elaboration of the PERFORM questionnaire, "Perceptions of Fatigue in Cancer Patients", there was no known instrument for evaluating Cancer-Related asthenia that had been developed and fully validated in Spain, which meant that certain aspects, relevant to our oncologic patients would not be being measured at the questionnaires from other contexts so cioculturales. These questionnaires reflect and evaluate a certain concept of quality of life related to health (HRQOL) that may not fit with the reality of Spanish patients, because the concept of HRQOL may differ depending on the cultural environment.

Questionnaire PERFORMS us to good viability: the average number of lost items per patient was less than 1 and $80 \%$ of patients completed to thickness of the questionnaire. The average time required for administration of the cues tionnaire was less than 9 minutes. Over $80 \%$ of patients considered the question easy or very easy to complete. The extreme scores were very Infer count $(<5 \%)$, this being an indication that the cues tionnaire covers well the intensity range of the symptom of the sample.

The internal consistency of me did was very satisfactory, both globally and by dimensions with an overall Cronbach alpha value of 0.935 .

The test-retest reliability in group of patients defined as stable was also very satisfactory, both overall dimensions as an intraclass correlation coefficient 0.832 overall.

In addition, the questionnaire present ta solid evidence of validity so that correlates consistently with health measures duly sarrolladas and validated, as the questions also relational consistently with variables related to the health, as Karnofsky index, the in density asthenia measured by visual analog scale or level of care required. Additionally it was significantly associated with levels have myoglobin of the patient $(\mathrm{P}=0.0163)$.

Finally, both the overall score and the scores of the di mansions of the questionnaire were PERFORM sensitive to changes in self-perceived health status by pa enough, especially when this change meant a worsening.

It is a tool that has proven to be feasible, reliable, valid and sensitive to change, and whose extension short (s or the 12 items) makes it very adequate for use in clinical practice is specialized, one of the variables that had us Keep in mind when choosing a measuring instrument, in order to avoid that the patient feels "assaulted" before so much evaluation.

The items of the questionnaire are distributed in 3 dimensions: habitual activities ( 4 items), attitudes and beliefs (4 items) and physical limitations (4 items). What makes the questionnaire show us values not only on the incidence of the symptoms of asthenia itself, but also establishes parameters of functionality of the patients. This last piece of information 


\section{Annals of Physiotherapy \& Occupational Therapy}

is of great value to us as occupational therapists, which makes this instrument the ideal one to later elaborate our therapeutic performance.

\section{Occupational therapist intervention}

\section{The intervention will consist of the following:}

- Progressive mobilization of the patient: In the bedridden cancer patient based on the capacities present in the individual, the objective will be to begin mobilization focusing on restoring lost autonomy. Progressively and taking into account all the health problems present in it.

- Reeducation in activities of daily living (ADL): Based on the present symptoms, the patient must adapt their daily routines, their activities, to their capacity for effort. In that case, we as occupational therapists must carry out a specific re-education program for their daily activities.

- Individualized education: Energy saving techniques (APR).

- Restructuring of rest periods: Hygienic measures for sleep disorders in cancer patients.

- Use of the activity as a rehabilitation tool.

- Muscle toning program.

- Assess the need for a support product that facilitates patient mobilization.

\section{Hygienic Measures for Sleep Disorders in Cancer Patients}

- Maintain a sleep-wake rhythm as regular as possible, especially when waking up.

- Avoid stimulating medication and other substances before sleeping.

- Maintain good pain control at night, preferably with long-life pain relievers.

- Avoid spending unnecessary time in bed during the day; for bedridden patients, providing physical and cognitive stimuli throughout the day.

- Minimize nighttime interruptions due to noise, medication administration or other environmental conditions.

- Remove unpleasant stimuli, such as clocks, from the room.

- Avoid being in bed during the night awake, trying to sleep; Do some relaxing activity (reading), preferably out of bed, until drowsiness appears.

- Avoid naps in the late afternoon.

- Use hypnotic medication after a correct evaluation of the sleep disorder and avoid its overuse.

\section{References}

1. Barco E, Sanchez F (2008) Continuous care concept. History, principles and bases of palliative medicine. General principles of symptom control. The subcutaneous route In: Cruz JJ, Rodriguez CA, Barco E, et al. (Eds.), Clinical Oncology. $4^{\text {th }}$ (Edn.), Madrid: Nova Sidonia, pp: 217-38.

2. Cooper J (2007) Challenges faced by occupational therapist in oncology and palliative care. In: Cooper $\mathrm{J}$, et al. (Eds.), Occupational therapy in oncology and palliative care. $2^{\text {nd }}($ Edn.), Londres, Jhon Wiley, pp: 11-27.

3. IARC (2010) Global cancer observatory. WHO.

4. Gonzalez M, Ordonez A (2004) La astenia tumoral. $1^{\text {st }}$ (Edn.), Madrid: Panamericana, pp: 9.

5. (2013) National Cancer Institute.

6. Sanchez F, Barco E (2008) Control of more prevalent symptoms: emesis and other digestive complications, asthenia, cachexia, other symptoms. Care at the end of life. In: Cruz JJ, Rodríguez CA, Barco E, et al. (Eds.), Clinical Oncology. $4^{\text {th }}$ (Edn.), Madrid: Nova Sidonia, pp: 299.

7. Wagner LI, Cella D (2004) Fatigue and cancer: Causes, prevalence and treatment approaches. British journal of Cancer 91(5): 822-828.

8. Rodriguez MA, Lizon J (2004) Concept and etiopathogenesis of asthenia. In: Gonzalez M, Ordonez A, et al. (Eds.), Tumor asthenia. $1^{\text {st }}$ (Edn.), Madrid: Panamericana, pp: 1-11.

9. Baron MG, Ordonez A (2004) Tumor asthenia: a poorly studied syndrome. Psychooncology: biopsychosocial research and clinic in oncology. 1(2): 25-28.

10. Rodriguez CA, Ruiz M, Alonso R, Viotti F, Del Barco E, et al. (2012) Assessment of asthenia in oncology. Application of the Perform Questionnaire 9(1): 65-79.

11. Cruz JJ, Rodriguez CA, Del Barco E (2008) Cilinical oncology. $4^{\text {th }}$ (Edn.), In: Sidonia N, et al. (Eds.), 17: 299302.

12. Ordonez A, Gonzalez M, Feliu J (2001) Supportive treatment in the cancer patient. Some proposals based on our experience. Rev Oncologia 3: 266-269.

13. Espinosa E, Zamora P (2007) Quality of life. In: Baron MG, et al, (Eds.), Palliative medicine and support treatment in the cancer patient. $2^{\text {nd }}(E d n)$, Madrid: Panamericana, pp: 43-51.

14. Kielhofner G (2006) Occupational therapy practice. In: Kielhofner G, et al. (Eds.), Conceptual foundations of occupational therapy. $1^{\text {st }}$ (Edn.), Buenos Aires: Panamericana, pp: 4-6. 


\section{Annals of Physiotherapy \& Occupational Therapy}

15. Cooper J (1997) Occupational therapy in oncology and palliative care. Wiley, pp: 284.

16. Mock V, Atkinson A, Barsevick A, Cella D, Cimprich B, et al. (2000) NCCN practice guidelines for cancer-relatedfatigue. Oncology (Williston Park) 14(11A): 151-161.
17. Fernandez EJ, Rihuete MI, Cruz JJ (2018) Randomized study on the influence of a comprehensive rehabilitation program on control of cancer-related asthenia. Randomized study on the influence of a comprehensive rehabilitation program on control of cancer-related asthenia. 25(3): 160-167. 